\title{
FUNGICIDAS E MISTURA DE FUNGICIDAS NO CONTROLE DO OÍDIO DA SOJA
}

\author{
LUIZ E. B. BLUM ${ }^{1}$, EMERSON F. REIS ${ }^{1}$, ALEXANDER G. PRADE ${ }^{2} \&$ VANDERLEI J. TAVELA ${ }^{2}$
}

${ }^{1}$ Departamento de Fitotecnia, Centro de Ciências Agroveterinárias, Universidade do Estado de Santa Catarina, Cx. Postal 81, 88520-000, Lages, SC, e-mail: a2lbb@cav.udesc.br; ${ }^{2}$ Milênia Agro Ciências S.A., Londrina, PR

(Aceito para publicação em 14/11/2001)

Autor para correspondência: Luiz E. B. Blum

BLUM, L.E.B., REIS, E.F., PRADE, A.G. \& TAVELA, V.J. Fungicidas e mistura de fungicidas no controle do oídio da soja. Fitopatologia Brasileira 27:216-218. 2002.

\begin{abstract}
RESUMO
Dois experimentos foram conduzidos em Londrina/PR para avaliar a eficácia de fungicidas e suas misturas no controle do oídio (Microsphaera diffusa) da soja (Glycine max). Uma aplicação dos produtos foi feita no estádio R4 da soja cv. EMBRAPA 48. Os melhores resultados foram obtidos com os seguintes fungicidas: tebuconazol-1 e tebuconazol-2, carbendazim-1 e carbendazim-2 (produtos comerciais

diferentes). Teste 1 - tebuconazol-2 (150 g i. a./ha), prochloraz + propiconazol $(225+62,5)$, propiconazole + carbendazim-1 $(50+175)$ e tebuconazol-1 + propiconazol $(75+62,5)$; Teste 2 - carbendazim1 (250), carbendazim-2 (250) e tebuconazol-2 (100).

Palavras-chave adicionais: Glycine max, Microsphaera diffusa, oídio, cinza.
\end{abstract}

\section{ABSTRACT \\ Fungicides and fungicide mixtures to control soybean powdery mildew}

Two experiments were performed in Londrina, PR, Brazil to evaluate the efficacy of fungicides on the control of soybean (Glycine max) powdery mildew (Microsphaera diffusa). One application of the different commercial products was made on soybean cv. EMBRAPA 48 growth stage $\mathrm{R} 4$. The best fungicides to control powdery mildew were tebuconazole- 1 and tebuconazole-2, carbendazin-1 and carbendazin-2. Test 1 - tebuconazole-2 (150 g a.i. / ha), prochloraz + propiconazole $(225+62.5)$, propiconazole + carbendazin-1 $(50+175)$, and tebuconazole- $1+$ propiconazole $(75+62.5)$; Test 2 - carbendazin1 (250), carbendazin-2 (250), and tebuconazole-2 (100).
O oídio da soja (Glycine max Merrill) causado por Microsphaera diffusa Cooke \& Peck, embora considerado de pouca importância há alguns anos (Yorinori, 1997), ultimamente vem aumentando em prevalência. A partir de 1996/1997, desde a região Sul até as regiões Sudeste e Centro Oeste do Brasil, foram relatados diversos surtos epidêmicos desta doença (Reis et al., 1997; Sawada \& Azevedo, 1997; Michel et al., 1998). Algumas vezes, nos casos de elevada colonização dos tecidos superficiais da planta por oídio, é possível ocorrer uma redução significativa no rendimento da soja devido à redução da área fotossinteticamente ativa.

A infecção da soja por oídio é favorecida por temperaturas em torno de $20{ }^{\circ} \mathrm{C}$ (McGee, 1992), média a alta umidade relativa do ar (50-90\%), baixa incidência e intensidade de precipitação e quando as plantas encontramse entre os estágios R1 (início da floração) e R6 (formação completa de sementes). Cada ciclo desta doença, sob condições favoráveis à mesma, dura cerca de sete a dez dias (Picinini \& Fernandes, 1998).

Existem cultivares resistentes (Reis et al, 1997; Tanaka et al., 1997), porém muitas delas não são recomendadas ou não estão disponíveis para o plantio em determinadas regiões brasileiras. Portanto, uma das formas de controlar a doença na ausência de cultivares resistentes ou na impossibilidade do uso das mesmas é através da aplicação preventiva de fungicidas. Entre os fungicidas tradicionalmente aplicados contra o oídio citam-se o enxofre elementar, tiabendazol, benomil, tiofanato metílico e o clorotalonil (McGee, 1992). Nos últimos anos vários outros fungicidas são utilizados para o controle do oídio nas mais diversas culturas, como o difenoconazol, tebuconazol, propiconazol, procloraz e o carbendazim (Picinini \& Fernandes, 1997; Sawada \& Azevedo, 1997). Em função destas informações desenvolveram-se em campo dois testes com o objetivo de avaliar o efeito de diferentes doses, diferentes misturas e de diferentes fungicidas sobre a incidência e severidade do oídio e sobre o rendimento da soja.

Dois ensaios foram realizados em Londrina/PR, utilizando a cultivar EMBRAPA 48 plantada em 20/10/1999 em um Latossolo roxo previamente arado, corrigido (fertilização e calagem) e gradeado. A densidade de plantio foi de 300 mil plantas/ha (14 sementes/m e espaçamento de 45 $\mathrm{cm}$ entre linhas). Todos os tratos culturais (inoculação de sementes com Bradyrrhizobium, aplicação de herbicidas e inseticidas) da soja foram efetuados conforme o padrão para a região, exceto o uso de fungicidas. Nos ensaios apenas uma pulverização dos produtos foi efetuada quando a soja encontrava-se no estádio R4 (formação completa de vagens) 
de desenvolvimento (Ritchie et al., 1982) em 21/1/2000. Utilizou-se um pulverizador costal de barra com aspersor pressurizado $\left(\mathrm{CO}_{2}\right)$ de precisão $(2,8$ bar $)$ que distribuía o equivalente a 200 1/ha de calda. A barra de pulverização possuía um bico (TXVS08) a cada $25 \mathrm{~cm}$, totalizando nove bicos.

Os experimentos foram delineados em blocos ao acaso com nove tratamentos e quatro repetições. Cada unidade experimental dos ensaios constituiu-se de uma parcela com $16 \mathrm{~m}^{2}(4 \times 4 \mathrm{~m})$ de área total e $8 \mathrm{~m}^{2}$ de área útil para avaliação. Os dados coletados de incidência, severidade e de rendimento de cada tratamento foram avaliados estatisticamente (Teste $\mathrm{F}, \mathrm{P}<5 \%$ ) através de análise de variância e se comprovada a significância das médias de dados dos tratamentos, as mesmas foram comparadas entre si pelo teste de Tukey $(\mathrm{P}=5 \%)$.

Nos ensaios 1 e 2 foram feitas quatro avaliações (29/1, 4/2, 10/2 e 22/2) de incidência e severidade de oídio entre os estágios R5 e início do R7 (início da maturação) de desenvolvimento da soja (Ritchie et al., 1982). Em cada uma das parcelas coletou-se aleatoriamente de 15 plantas um trifolíolo do terço médio e superior da planta, avaliando-se então a incidência $(\%)$ e a severidade (\% de área foliar afetada) conforme escala diagramática apresentada por Azevedo (1998) para avaliação da severidade do oídio (Erysiphe polygoni DC.) do feijoiro (Phaseolus vulgaris L.). A colheita de vagens em ambos experimentos foi feita em 30/3/2000.

Os produtos avaliados nos testes foram o carbendazim (nome comercial - 1: Bendazol, suspensão concentrada, $500 \mathrm{~g}$ de ingrediente ativo (ia)/1; nome comercial - 2: Derosal, SC, $500 \mathrm{~g}$ ia/l), prochloraz (nome comercial: Jade, concentrado emulsionável, $450 \mathrm{~g} / 1$ ), propiconazol (nome comercial: Juno, CE, $250 \mathrm{~g} / \mathrm{l}$ ) e tebuconazol (nome comercial - 1: Orius $250 \mathrm{CE}, 250 \mathrm{~g} / \mathrm{l}$; nome comercial - 2: Folicur $200 \mathrm{CE}, 200 \mathrm{~g} / \mathrm{l}$ ), de acordo com os tratamentos aplicados nos dois ensaios (Tabela 1). As testemunhas experimentais foram pulverizadas com água.

Em muitas situações, o controle de doenças foliares através da aplicação de fungicidas é uma das poucas alternativas. Os fungicidas pertencentes aos grupos dos benzimidazois e dos triazois estão entre os mais aplicados para o controle de manchas foliares, ferrugens e oídios da soja e de outras culturas (Blum \& Gabardo, 1993). Os resultados dos experimentos aqui apresentados mostram a redução da severidade e incidência do oídio da soja através do uso de alguns fungicidas pertencentes aos grupos supra citados à exceção do procloraz (imidazol).

No primeiro ensaio (Tabela 1) todos os fungicidas foram igualmente eficientes na redução da severidade do

TABELA 1 - Efeito de fungicidas na incidência e severidade de oídio (Microsphaeria diffusa) e no rendimento de grãos de soja (Glycine max)

\begin{tabular}{|c|c|c|c|c|c|c|c|c|c|}
\hline \multirow{2}{*}{$\begin{array}{l}\text { Experimento } 1 \\
\text { g/ha de i. a. }\end{array}$} & \multicolumn{4}{|c|}{ Incidência (\%) } & \multicolumn{4}{|c|}{$\begin{array}{c}\text { Severidade } \\
\text { (\% de área foliar afetada) } \\
\end{array}$} & \multirow{2}{*}{$\begin{array}{l}\mathrm{kg} / \mathrm{ha} \\
\times 1000\end{array}$} \\
\hline & 1 & 2 & 3 & 4 & 1 & 2 & 3 & 4 & \\
\hline Procloraz 225 + Propiconazol 62,5 & $96,67 \mathrm{a}$ & $86,67 \mathrm{ab}$ & $50,00 \mathrm{ab}$ & $69,99 \mathrm{ab}$ & $5,90 \mathrm{a}$ & $2,79 a$ & $1,46 \mathrm{a}$ & $2,38 \mathrm{a}$ & $3,52 b$ \\
\hline Tebuconazol-1 75 + Procloraz 225 & $100,00 \mathrm{a}$ & $80,00 \mathrm{ab}$ & $36,67 \mathrm{a}$ & $66,66 \mathrm{a}$ & $8,37 \mathrm{a}$ & $2,75 \mathrm{a}$ & $1,08 \mathrm{a}$ & $1,36 \mathrm{a}$ & $2,99 b$ \\
\hline Procloraz 225 & $100,00 \mathrm{a}$ & $68,34 \mathrm{ab}$ & $35,00 \mathrm{a}$ & $80,00 \mathrm{ab}$ & $5,28 \mathrm{a}$ & $2,17 \mathrm{a}$ & $0,82 \mathrm{a}$ & $2,10 \mathrm{a}$ & $2,79 \mathrm{ab}$ \\
\hline Tebuconazol-1 50 & $98,33 \mathrm{a}$ & $78,33 \mathrm{ab}$ & $40,00 \mathrm{a}$ & $71,00 \mathrm{ab}$ & $7,63 \mathrm{a}$ & $2,14 \mathrm{a}$ & $0,88 \mathrm{a}$ & $1,76 a$ & $2,90 \mathrm{~b}$ \\
\hline Tebuconazol-2 150 & $100,00 \mathrm{a}$ & $55,00 \mathrm{a}$ & $53,33 \mathrm{ab}$ & $61,00 \mathrm{a}$ & $7,35 \mathrm{a}$ & $1,34 \mathrm{a}$ & $1,02 \mathrm{a}$ & $1,81 \mathrm{a}$ & $3,74 b$ \\
\hline Tebuconazol-1 75 + Propiconazol 62,5 & $98,33 \mathrm{a}$ & $66,67 \mathrm{ab}$ & $28,33 \mathrm{a}$ & $53,33 \mathrm{a}$ & $6,68 \mathrm{a}$ & $1,85 \mathrm{a}$ & $0,70 \mathrm{a}$ & $1,56 \mathrm{a}$ & $2,72 \mathrm{ab}$ \\
\hline Tebuconazol-1 62,5 + Carbendazim-1 175 & $98,33 \mathrm{a}$ & $63,34 \mathrm{a}$ & $38,33 \mathrm{a}$ & $58,33 \mathrm{a}$ & $7,60 \mathrm{a}$ & $2,02 \mathrm{a}$ & $1,20 \mathrm{a}$ & $1,36 \mathrm{a}$ & $2,96 b$ \\
\hline Propiconazol 50 + Carbendazim-1 175 & $96,67 \mathrm{a}$ & $75,00 \mathrm{ab}$ & $50,00 \mathrm{ab}$ & $75,00 \mathrm{ab}$ & $8,99 \mathrm{a}$ & $2,02 \mathrm{a}$ & $1,20 \mathrm{a}$ & $1,93 \mathrm{a}$ & $3,48 b$ \\
\hline Testemunha & $100,00 \mathrm{a}$ & $100,00 \mathrm{~b}$ & $100,00 \mathrm{~b}$ & $100,00 \mathrm{~b}$ & $26,59 b$ & $7,00 \mathrm{~b}$ & $7,10 \mathrm{~b}$ & $24,28 b$ & $2,00 \mathrm{a}$ \\
\hline C. V. $(\%)$ & 3,23 & 18,01 & 33,20 & 18,15 & 36,89 & 44,61 & 42,20 & 39,06 & 18,84 \\
\hline \multicolumn{10}{|l|}{ Experimento 2} \\
\hline Carbendazim-1 250 & $95,00 \mathrm{a}$ & $65,00 \mathrm{ab}$ & $45,00 \mathrm{ab}$ & $98,33 b$ & $4,50 \mathrm{a}$ & $2,17 \mathrm{a}$ & $1,45 \mathrm{a}$ & $2,38 \mathrm{ab}$ & $3,62 b$ \\
\hline Propiconazol 125 & $93,33 \mathrm{a}$ & $70,00 \mathrm{ab}$ & $38,33 \mathrm{ab}$ & $51,67 \mathrm{a}$ & $5,27 \mathrm{a}$ & $2,27 \mathrm{a}$ & $1,32 \mathrm{a}$ & $1,36 \mathrm{a}$ & N.D. \\
\hline Propiconazol 75 & $91,67 \mathrm{a}$ & $50,00 \mathrm{a}$ & $25,00 \mathrm{a}$ & $65,00 \mathrm{ab}$ & $4,13 \mathrm{a}$ & $2,03 \mathrm{a}$ & $0,88 \mathrm{a}$ & $2,10 \mathrm{a}$ & $3,36 \mathrm{ab}$ \\
\hline Tebuconazol-1 100 & $95,00 \mathrm{a}$ & $65,00 \mathrm{ab}$ & $36,67 \mathrm{ab}$ & $46,67 \mathrm{a}$ & $5,62 \mathrm{a}$ & $1,97 \mathrm{a}$ & $1,34 \mathrm{a}$ & $1,76 \mathrm{a}$ & N.D. \\
\hline Tebuconazol-1 100 + Carbendazim-1 150 & $91,67 \mathrm{a}$ & $45,00 \mathrm{a}$ & $28,33 \mathrm{a}$ & $58,33 \mathrm{ab}$ & $4,47 \mathrm{a}$ & $1,65 \mathrm{a}$ & $1,04 \mathrm{a}$ & $1,81 \mathrm{a}$ & $2,93 \mathrm{ab}$ \\
\hline Tebuconazol-2 100 & $96,67 \mathrm{a}$ & $68,33 \mathrm{ab}$ & $35,00 \mathrm{ab}$ & $60,00 \mathrm{ab}$ & $4,22 \mathrm{a}$ & $2,04 \mathrm{a}$ & $0,95 \mathrm{a}$ & $1,56 \mathrm{a}$ & $3,48 b$ \\
\hline Carbendazim-2 250 & $98,33 \mathrm{a}$ & $80,00 \mathrm{ab}$ & $51,67 \mathrm{ab}$ & $80,00 \mathrm{ab}$ & $5,82 \mathrm{a}$ & $2,52 \mathrm{a}$ & $1,78 \mathrm{a}$ & $1,36 \mathrm{a}$ & $3,53 b$ \\
\hline Procloraz 337,5 & $88,33 \mathrm{a}$ & $71,67 \mathrm{ab}$ & $41,67 \mathrm{ab}$ & $75,00 \mathrm{ab}$ & $4,45 \mathrm{a}$ & $2,55 \mathrm{a}$ & $1,27 \mathrm{a}$ & $1,93 \mathrm{a}$ & $3,42 \mathrm{ab}$ \\
\hline Testemunha & 100,00 & $100,00 b$ & $100,00 b$ & $100,00 b$ & $25,50 \mathrm{~b}$ & $10,73 b$ & $13,92 b$ & $28,54 b$ & $2,61 \mathrm{a}$ \\
\hline C. V. $(\%)$ & 9,37 & 22,64 & 42,10 & 23.33 & 35,00 & 47,47 & 75,73 & 51,08 & 18,83 \\
\hline
\end{tabular}

Médias seguidas pela mesma letra na mesma coluna não diferem (Tukey 5\%); N.D. = não determinado. 


\section{L.E. Blum et al.}

oídio. Nas quatro avaliações efetuadas os tratamentos com fungicidas diferiram significativamente da testemunha sem fungicidas. Nos tratamentos com procloraz + propiconazol, tebuconazol-1 + procloraz, tebuconazol-1, tebuconazol-2, tebuconazol-1 + carbendazim-1 e propiconazol + carbendazim-1 obteve-se uma maior produtividade da soja. Constatou-se que a incidência do oídio não foi afetada de início, porém na terceira e quarta avaliações houve uma significativa redução de incidência em vários tratamentos com fungicidas em relação à testemunha. Milleo et al. (1999) informaram que misturas entre fentin hidróxido e carbendazim ou procloraz foram eficientes no controle do oídio da soja em Ponta Grossa, PR.

No segundo experimento houve redução significativa da severidade de oídio da soja nos tratamentos onde foram aplicados os fungicidas. A incidência da doença apresentou-se significativamente reduzida da segunda a quarta avaliações nos tratamentos com os produtos carbendazim-1, propiconazol, tebuconazol-1 e tebuconazol$1+$ carbendazim-1. A produtividade da soja foi significativamente maior onde foram aplicados tebuconazol-2, carbendazim-1 e carbendazim-2. Os tratamentos com tebuconazol-1 e tebuconazol-2 não diferiram entre si quanto a redução da severidade e incidência do oídio. As médias de produtividade dos tratamentos com propiconazol (125) e Tebuconazol-1 foram omitidas pela mistura involuntária de paralelas entre os referidos tratamentos. Michel \& Reis (1998) relataram que os fungicidas propiconazol e tebuconazol destacaram-se no controle do oídio e do complexo de doenças de final de ciclo da soja em Não-me-toque, RS, porém não influenciaram significativamente a produtividade. Em Rolândia., PR, o difenoconazol e a mistura de difenoconazol + propiconazol foram os melhores no controle do oídio da soja (Utiamada et al., 1999). Sawada \& Azevedo (1997) relataram a eficiência do propiconazol e da mistura propiconazol + difenoconazol no controle do oídio em Cascavel, PR.

As misturas de fungicidas foram igualmente eficientes na redução do oídio quando comparadas aos produtos aplicados isoladamente. Contudo, como vantagem, as misturas reduzem a possibilidade da seleção de raças resistentes do patógeno aos fungicidas e contribuem com o controle de outras doenças da soja. No presente estudo, a mistura tebuconazol + procloraz e o propiconazol (75) destacaram-se na redução do oídio e na contribuição ao aumento da produtividade da soja. Salientaram-se ainda, carbendazim-1, carbendazim-2, tebuconazol-2, tebuconazol-1 + carbendazim-1 e propiconazol + carbendazim-1.

\section{REFERÊNCIAS BIBLIGRÁFICAS}

AZEVEDO, L.A.S. Manual de quantificação de doenças de plantas. São Paulo, Novartis Biociências - Setor Agro, 1998.

BLUM, L.E.B. \& GABARDO, H. Controle químico da ferrugem do alho na região de Curitibanos/SC. Fitopatologia Brasileira 18:230-232. 1993.

McGEE, D.C. Soybean diseases: a reference source for seed technologists. St. Paul, APS Press, 1992.

MICHEL, C.A. \& REIS, E.M. Controle químico do oídio e do complexo de doenças de final de ciclo na cultura da soja. Fitopatologia Brasileira 23:260. 1998. (Resumo)

MICHEL, C.A., REIS, E.M. \& VIEIRA, R. Controle químico do oídio na cultura da soja. Fitopatologia Brasileira 23:259. 1998. (Resumo)

MILLEO, M.V.R., VENANCIO, W.S., ZAGONEL, J. \& CASTANHEIRAS, A.F.F. Avaliação de fentin hydroxide aplicado isoladamente e em mistura no controle do oídio, induzido por Microsphaera diffusa em soja. Fitopatologia Brasileira 24:306. 1999. (Resumo)

PICININI, E.C. \& FERNANDES, J.M. Controle químico do oídio Microsphaera diffusa em soja no ano de 1997. Fitopatologia Brasileira 22:297. 1997. (Resumo)

PICININI, E.C. \& FERNANDES, J.M. Doenças da soja: diagnose, epidemiologia e controle. Passo Fundo, EMBRAPA-Trigo, 1998.

REIS, E.M., MEDEIROS, C.A. \& CASA, R.T. Epidemia de oídio da soja, causada por Microsphaera diffusa, na safra 1996/ 97, no RS. Fitopatologia Brasileira 22:300-301. 1997. (Resumo)

RITCHIE, S., HANWAY, J.J. \& THOMPSON, H.E. How a soybean plant develops. Ames: Iowa State University of Science and Technology, Coop. Ext. Serv., Special Report, 53. 1982.

SAWADA, E. \& AZEVEDO, L.A.S. Avaliação de fungicidas no controle do oídio (Erysiphe polygoni DC.) da soja. Fitopatologia Brasileira 22:306. 1997. (Resumo)

TANAKA, M.A.S., MASCARANHAS, M.A.A. \& ITO, M.F. Reação de cultivares de soja ao oídio (Microsphaera diffusa). Fitopatologia Brasileira: 22:314. 1997. (Resumo)

UTIAMADA, C.M., SATO, L.N., VIDA, J.B. \& YORINORI, J.T. Eficiência de fungicidas no controle de oídio da soja. Fitopatologia Brasileira 24:339-340. 1999. (Resumo)

YORINORI, J.T. Soja (Glycine max (L.) Merril) - controle de doenças. In: Ribeiro do Vale, F.X. \& Zambolim, L. (Eds.) Controle de doenças de plantas. V. 2. Viçosa, UFV/MAA, 1997. Cap. 21, pp.953-1023. 